\title{
Letter Report: Looking Ahead at Nuclear Fuel Resources
}

J. Stephen Herring

September 2013

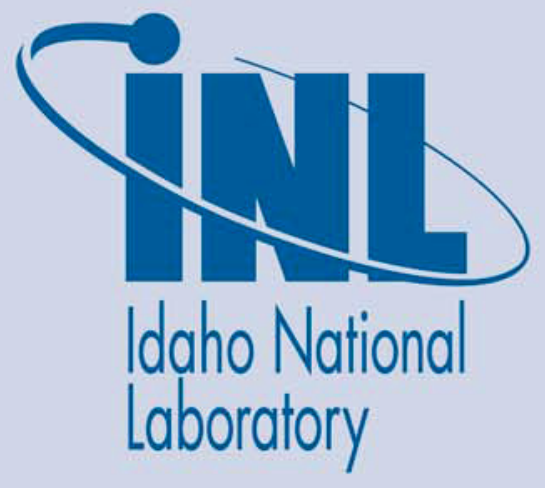

The INL is a U.S. Department of Energy National Laboratory operated by Battelle Energy Alliance 


\section{DISCLAIMER}

This information was prepared as an account of work sponsored by an agency of the U.S. Government. Neither the U.S. Government nor any agency thereof, nor any of their employees, makes any warranty, expressed or implied, or assumes any legal liability or responsibility for the accuracy, completeness, or usefulness, of any information, apparatus, product, or process disclosed, or represents that its use would not infringe privately owned rights. References herein to any specific commercial product, process, or service by trade name, trade mark, manufacturer, or otherwise, does not necessarily constitute or imply its endorsement, recommendation, or favoring by the U.S. Government or any agency thereof. The views and opinions of authors expressed herein do not necessarily state or reflect those of the U.S. Government or any agency thereof. 


\title{
Letter Report: Looking Ahead at Nuclear Fuel Resources
}

\author{
J. Stephen Herring
}

September 2013

\begin{abstract}
Idaho National Laboratory
Nuclear Systems Design and Analysis

Idaho Falls, Idaho 83415
\end{abstract}

http://www.inl.gov

Prepared for the

U.S. Department of Energy

Office of Nuclear Energy

Under DOE Idaho Operations Office

Contract DE-AC07-05ID14517 


\begin{abstract}
The future of nuclear energy and its ability to fulfill part of the world's energy needs for centuries to come depend on a reliable input of nuclear fuel, either thorium or uranium. Obviously, the present nuclear fuel cycle is completely dependent on uranium. Future thorium cycles will also depend on ${ }^{235} \mathrm{U}$ or fissile isotopes separated from used fuel to breed ${ }^{232} \mathrm{Th}$ into fissile ${ }^{233} \mathrm{U}$.

This letter report discusses several emerging areas of scientific understanding and technology development that will clarify and enable assured supplies of uranium and thorium well into the future.

At the most fundamental level, the nuclear energy community needs to appreciate the origins of uranium and thorium and the processes of planetary accretion by which those materials have coalesced to form the earth and other planets. Secondly, the studies of geophysics and geochemistry are increasing understanding of the processes by which uranium and thorium are concentrated in various locations in the earth's crust. Thirdly, the study of neutrinos and particularly geoneutrinos (neutrinos emitted by radioactive materials within the earth) has given an indication of the overall global inventories of uranium and thorium, though little indication for those materials' locations. Crustal temperature measurements have also given hints of the vertical distribution of radioactive heat sources, primarily ${ }^{238} \mathrm{U}$ and ${ }^{232} \mathrm{Th}$, within the continental crust. Finally, the evolving technologies for laser isotope separation are indicating methods for reducing the energy input to uranium enrichment but also for tailoring the isotopic vectors of fuels, burnable poisons and structural materials, thereby adding another tool for dealing with long-term waste management.
\end{abstract}




\section{Table of Contents}

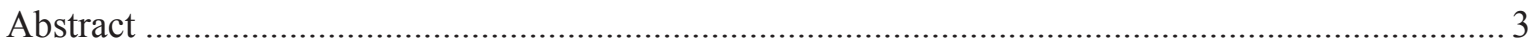

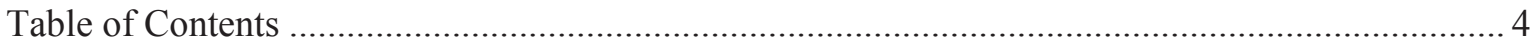

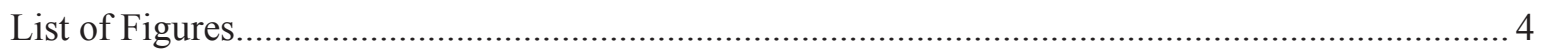

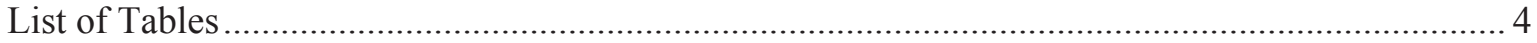

Fundamental understanding of the astrophysical origins of heavy elements .................................. 5

Mechanisms for the Concentration of Uranium ........................................................................ 7

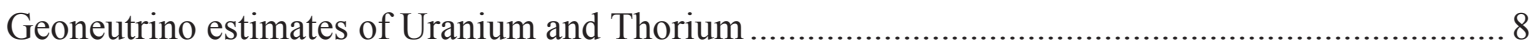

Average Vertical Distribution of Uranium and Thorium in the Crust......................................... 12

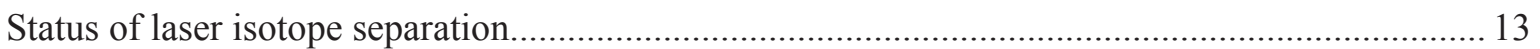

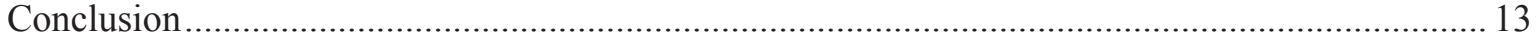

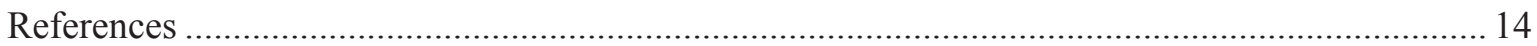

\section{List of Figures}

Figure 1. Isotopic distribution $0.48 \mathrm{~s}$ after Type II Supernova collapse ........................................ 6

Figure 2. Isotopic distribution $0.58 \mathrm{~s}$ after Type II Supernova collapse ........................................ 6

Figure 3. Isotopic distribution $2.13 \mathrm{~s}$ after Type II Supernova collapse …..................................... 7

Figure 4. Global U + Th Content based on KAMLAND Geoneutrino Data .................................. 11

\section{List of Tables}



Table $2 \mathrm{U}$, Th and $\mathrm{K}$ global inventories, radiogenic heating and neutrino luminosities according to the Bulk Silicate Earth (BSE) model ..................................................................... 10

Table 3. Sources of Heat in the Upper Continental Crust .......................................................... 12

Table 4. Temperature Distribution Depth Parameter .................................................................. 12

Table 5. Comparison of Enrichment Methods ..................................................................................... 13 


\section{Fundamental understanding of the astrophysical origins of heavy elements}

Uranium, thorium and all other elements heavier than nickel result from the sudden collapse of massive stars as supernovae. The lifetime of stars and the results of these gravity-driven implosions are very dependent on the stars' initial mass. A star having the mass of our sun lasts for about 10 billion years but can only produce elements up to iron. A star having 10 solar masses lasts for only 10 million years until it implodes and then explodes as a supernova, producing all the elements in the periodic table.

The ground-breaking work by Burbidge, Burbidge, Fowler and Hoyle ${ }^{1}$ led to the realization that all of the elements heavier than nickel are the result of less than a minute of tremendous neutrino and neutron fluxes during the collapse and explosion of a supernova. ${ }^{2}$ During the last few minutes of such a massive star's evolution, hydrogen, helium and all of the elements lighter than nickel at the center of the star are depleted through fusion reactions. With no more energy available for continued fusion reactions, the center cannot withstand gravitational forces of the outer shells of material and the matter in the center is compressed to a degenerate state in which matter is broken into the constituent particles, primarily neutrons and neutrinos. The torrent of neutrons from the center of the supernova irradiates the in-falling outer layers of stellar material, producing heavier isotopes at a rate faster than the $\beta^{-}$decays of those isotopes. The result is the production of isotopes stretching from nickel through uranium and beyond, all saturated with neutrons.

The distribution of the elements and their isotopes as a function of time in a supernova has been simulated $^{3}$ and indicates that isotopes saturated with neutrons ("the neutron drip edge") form during a few seconds of intense activity at the center of the imploding supernova. From this nuclear modeling of a supernova explosion we can infer that uranium and thorium are about seven orders of magnitude below silicon in the composition of the supernova debris - the material from which planets are formed.

Earlier studies are also in agreement. Urey ${ }^{4}$ cites estimates by Goldschmidt of the primordial abundance of 41 weight parts per billion (wppb) for uranium and $106 \mathrm{wppb}$ for thorium. Alpher's theoretical curves ${ }^{5}$ and Harrison S. Brown's observed astrophysical data ${ }^{6}$ show uranium approximately 6.5 orders of magnitude less abundant than silicon, resulting in a primordial abundances of $57 \mathrm{wppb}$. Deffeyes, accounting for the decay of uranium since the expansion of the primordial neutron gas, estimates global uranium abundance at $10.5 \mathrm{wppb}^{7}$

This type of supernova explosion is estimated to occur, somewhere in the universe, at the rate of one per second. Obviously, most such explosions are too distant or masked by dust clouds and are not detected from the earth. Since the beginning of the universe, some interstellar material has gone through multiple cycles of collapse, explosion, dispersal, and accretion into new stars.

The hydrodynamic instabilities of the implosion result in a wide variation in the shapes of the resulting nebulae. Nevertheless, neutron transport and reaction codes have been developed to estimate the distribution of isotopes resulting from a supernova implosion. Wanajo and others ${ }^{8}$ have modeled the first few seconds of isotope production and show that the uranium mass should be about seven orders of magnitude less than that of silicon. Three snapshots of the isotopic distribution at the core of a Type II supernova during the $2.5 \mathrm{~s}$ following freeze-out (when free neutrons are no longer present) are show in Figures 1-3. In these three figures, isotopes are indicated by the white dots and stable/long-lived isotopes are indicated with bold white dots. Neutron-poor isotopes above the line of stability are not shown. For 
reference the location of ${ }^{232} \mathrm{Th},{ }^{235} \mathrm{U}$ and ${ }^{238} \mathrm{U}$ are circled in red. In these figures, the parameter $\mathrm{Y}$ is the isotopic abundance compared to ${ }^{1} \mathrm{H}$ and its logarithm to the base ten is plotted.

Th and $U$

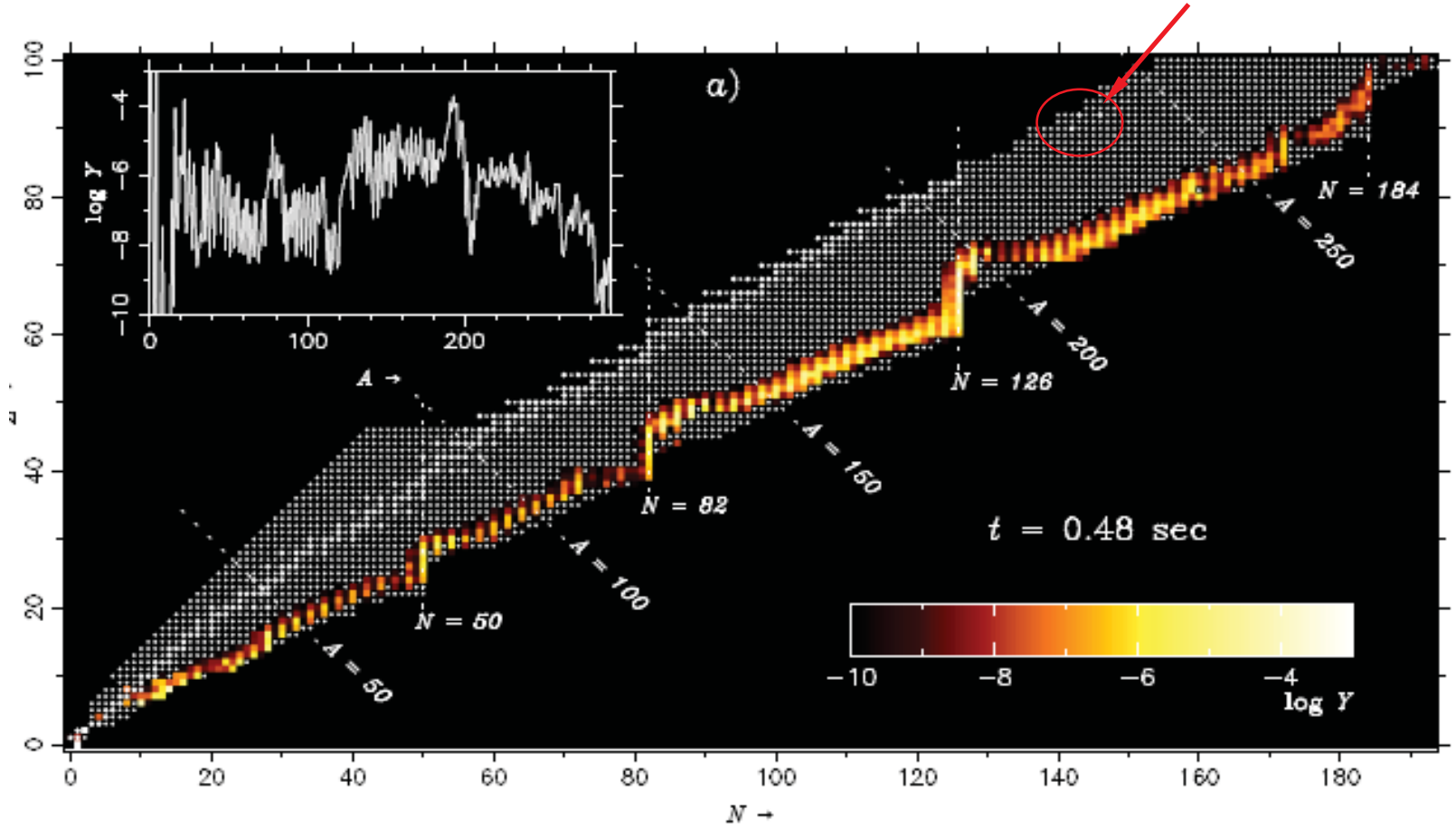

Figure 1. Isotopic distribution $0.48 \mathrm{~s}$ after Type II Supernova collapse

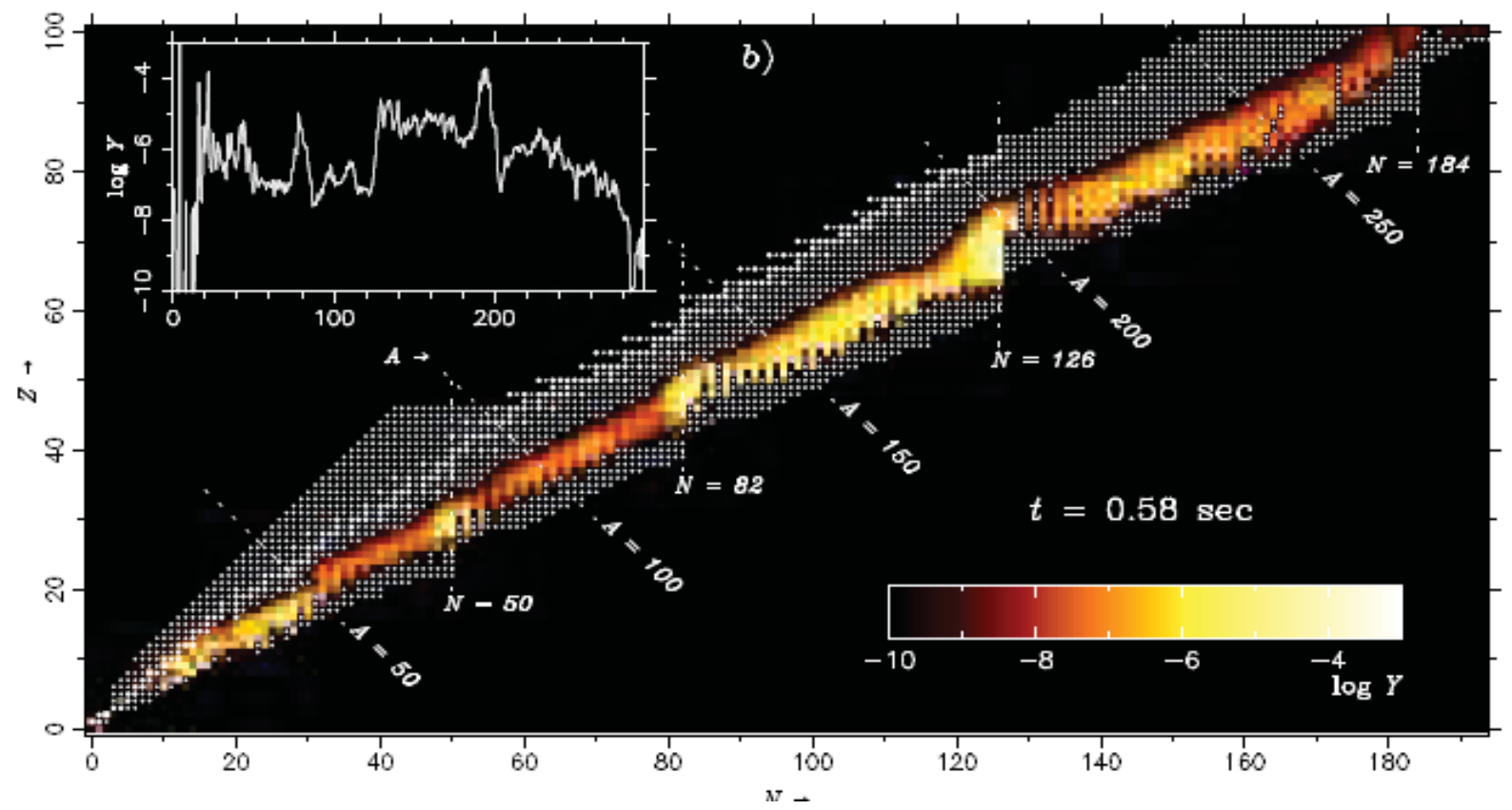

Figure 2. Isotopic distribution $0.58 \mathrm{~s}$ after Type II Supernova collapse 


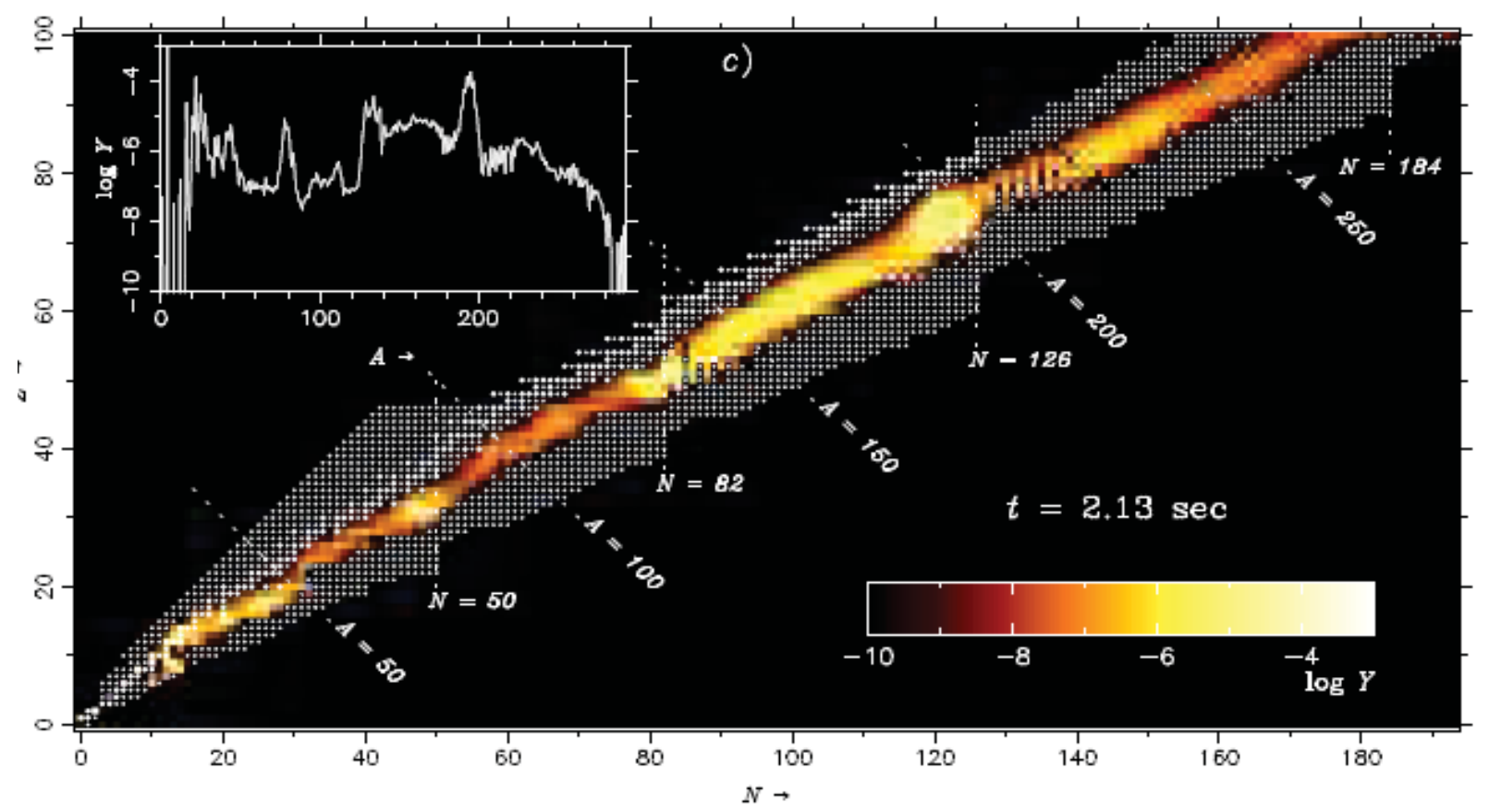

Figure 3. Isotopic distribution $2.13 \mathrm{~s}$ after Type II Supernova collapse

Since the chemical and planetary accretion characteristics of silicon, uranium and thorium are similar, and since the earth is about $10 \%$ silicon, one would expect that the overall concentration of uranium in the earth is about $10 \mathrm{wppb}$. As will be shown later, the geoneutrino data from KamLAND and from newer detectors indicate that the global uranium inventory is, in fact, about $10 \mathrm{wppb}$.

Therefore, based on these astrophysical models, it is fairly clear that the earth taken as uniform body contains about $10 \mathrm{wppb}$ uranium and about $40 \mathrm{wppb}$ thorium. Stated in other terms, the present global inventory is thus $\sim 60$ trillion metric tons $\left(60 \times 10^{12} \mathrm{t}\right)$ of uranium and $\quad 240 \quad \mathrm{x} \quad 10^{12} \mathrm{t}$ of thorium. Although this inventory is a vast amount of both elements, if uranium and thorium had a uniform distribution throughout the $\sim 6 \times 10^{21}$ metric tons of the earth, as assumed in the cold accretion model, concentrations of uranium and thorium would be far too small to be economically extracted.

\section{Mechanisms for the Concentration of Uranium}

Unlike other energy resources such as coal or petroleum, the resources of uranium are not fundamentally changed or lost by geological processes. Whereas petroleum might be lost through evaporation or combustion or a natural gas reservoir may vent into the atmosphere, uranium is lost only through radioactive decay or through the relatively rare formation of a natural reactor. Therefore the primordial inventory of uranium, reduced by radioactive decay, remains present somewhere in the earth. The crucial question is "where?".

The natural distribution of elements in the earth's crust is controlled by two major factors. The first is the set of ambient geological fractionating processes that leads to regions of depletion and concentration of the element. The second factor includes the overall geochemical characteristics of the element. Elements that are concentrated by a small number of fractionation processes can be expected to have a multi-modal distribution, with a peak in the tonnage versus grade curve for each of the modes of geochemical concentration. For elements having a large number of applicable concentration processes, the peaks overlap and the resulting tonnage versus grade curve takes on a log-normal characteristic. For example, the element chromium, whose distribution at high concentrations is solely governed by fractional 
crystallization in mafic magmas (i.e. high in magnesium and iron), one would expect a bimodal distribution of concentrations, with one peak at the average crustal abundance and the high concentration peak at the mafic fractionation concentration. On the other hand, most elements, uranium included, can undergo a wide variety of fractionation processes and deposits having a wide range of concentrations can be expected. In this latter case, the tonnage versus grade distribution would be expected to be log-normal. Bear in mind that geological conditions change over time and therefore the distribution patterns have varied with time.

In considering uranium in particular, it is important to examine the tectonic and igneous processes that have redistributed the uranium within the crust. In the past four billion years, the most important processes are continental accretion and plate tectonics. In the accretion process, crust formed into masses of continental dimensions. In the second, continuing, process, the continental crust and the oceanic crust have taken on quite different characteristics in terms of uranium concentration.

Igneous processes begin with the melting of mantle rocks at depths of 60 to 200 kilometers, followed by the migration of less dense liquids to the surface. The migration of these less dense minerals to the surface is a predominant process in the formation of the continental crust. The extruded liquid forms crust in two general locations, at mid-oceanic ridges, where the upwelling material forms new oceanic crust and in subduction zones, where the oceanic crust plunges back into the mantle, usually passing under the edge of a continent.

The behavior of uranium in igneous processes is dominated by two characteristics of the element. In the +4 oxidation state, the condition expected in the earth's mantle, the $\mathrm{U}^{+4}$ ion has an ionic radius of $97 \mathrm{x}$ $10^{12} \mathrm{~m}$ (picometers, pm) about the same as $\mathrm{Na}^{+1}$ ion $(97 \mathrm{pm})$. Other ions common in the core and mantle are significantly smaller in radius: $\mathrm{Fe}^{+2}, 74 \mathrm{pm} ; \mathrm{Ni}^{+2}, 69 \mathrm{pm}, \mathrm{Mg}^{+2}, 66 \mathrm{pm}$; and $\mathrm{Al}^{+3}, 51 \mathrm{pm}$. Thus, like sodium and the other large ions, uranium ions selectively enter partial melts within the mantle and are transported to the surface.

The second characteristic of uranium is its radioactivity, serving as a source of heat for melting the mantle and core. Like $\mathrm{Th}^{+4}$ (ionic radius $102 \mathrm{pm}$ ) and $\mathrm{K}^{+1}(133 \mathrm{pm}$ ), these heat-producing elements are readily fractionated out of the mantle and toward the surface. Deffeyes notes that the earth would be a radically different place if the heat-producing elements had small radii, since the geothermal energy source would then be located deep within the core and the convection currents driving plate tectonics would be much stronger. ${ }^{9}$

The rocks forming the oceanic crust at mid-oceanic ridges are characterized by a uniform uranium concentration of about $0.1 \mathrm{wppm}$. Conversely, the crust formed above subduction zones is characterized by uranium concentrations of about $2 \mathrm{wppm}$. The wide difference in concentration is due to the differences in the source materials and to the different chemistry. The upwelling mantle at the oceanic ridge has a uranium concentration of about $0.005 \mathrm{wppm}$, while the subduction zones have as their source material oceanic crust and bits of continental crust, with an average uranium concentration of about 0.1 wppm. The continuous upwelling at the oceanic ridges serves as a mechanism for depleting the core and mantle of uranium and incorporating that uranium in the oceanic crust. The relatively low concentration of uranium in the oceanic crust is augmented with uranium from continental runoff, which subsequently precipitates in the ocean basins. At the subduction zones, the oceanic crust is again subjected to partial melting and the uranium is again fractionated in the melt and transported to the surface.

\section{Geoneutrino estimates of Uranium and Thorium}

In the last twenty years a decay product of the 4.5 billion year-half-life of ${ }^{238} \mathrm{U}$ and the 14.2 billion yearhalf-life of ${ }^{232} \mathrm{Th}$, has been used to estimate the total global inventory of uranium and thorium. These 
particles, called neutrinos, are extremely difficult to detect and most neutrinos pass completely through the earth without interacting. Thus neutrino detectors are usually a thousand tons in mass and must be located deep underground to avoid unwanted signals caused by cosmic rays.

Neutrinos occur in three types: electron, muon and tau. Each of the three types has a corresponding antineutrino. Neutrinos originating within the earth, termed geoneutrinos, are actually electron anti-neutrinos primarily resulting from the decay of ${ }^{40} \mathrm{~K},{ }^{238} \mathrm{U}$ and ${ }^{232} \mathrm{Th}$. Geoneutrinos provide a means for estimating the total uranium and thorium content of the earth and also may provide very limited information on the location of those resources. These elementary particles have been measured over the past decade by massive detectors in Japan, Canada and Europe in an effort to differentiate the radiogenic and gravitational components of the total geothermal energy flux through the earth's surface. ${ }^{10}$ Neutrino and antineutrino fluxes have also been measured to understand neutrino oscillations, to investigate solar fusion processes and as a first signal of supernova events. Neutrinos (and anti-neutrinos) travel close to the speed of light, have a small mass $(<2 \mathrm{eV})$ and lack an electric charge. When an electron anti-neutrino collides with a proton, the result is a neutron and a positron (i.e. an anti-electron). This reaction, known as the neutron inverse $\beta$ decay, was used in the first detection of the neutrino in the Cowan-Reines experiment of 1956. ${ }^{11}$ Following the neutron inverse $\beta$ decay, the positron reacts with a nearby electron to produce two $511 \mathrm{keV}$ gamma rays. The neutron is absorbed by a hydrogen nucleus, releasing a characteristic $2.2 \mathrm{MeV}$ gamma with a mean delay of $\sim 200 \mu \mathrm{s}$. Circuitry in the detector registers a neutrino event through the delayed emission of a $2.2 \mathrm{MeV}$ gamma following two $511 \mathrm{keV}$ gammas.

The KamLAND (the Kamioka Large Anti-Neutrino Detector), in central Japan, consists of an 18-m diameter spherical vessel which in turn contains a 13-m diameter nylon balloon. The balloon contains approximately 1000 metric tons of a liquid scintillator (mineral oil, benzene and fluorescent compounds). The volume between the balloon and the spherical vessel contains highly purified oil which shields the balloon from external radiation and provides buoyancy to support the liquid scintillator. About 1900 photomultiplier tubes are mounted on the inner surface of the spherical vessel. Surrounding the spherical vessel is a water Cherenkov detector which provides additional shielding and acts as a muon veto counter.

Table 1 The main properties of geoneutrinos

\begin{tabular}{|c|c|c|c|c|c|}
\hline Decay & $\begin{array}{c}\mathrm{Q} \\
{[\mathrm{MeV}]}\end{array}$ & $\begin{array}{c}\tau_{1 / 2} \\
{\left[10^{9} \mathrm{yr}\right]}\end{array}$ & $\begin{array}{l}\mathrm{E}_{\max } \\
{[\mathrm{MeV}]}\end{array}$ & $\begin{array}{c}\varepsilon_{\mathrm{H}} \\
{[\mathrm{W} / \mathrm{kg}]}\end{array}$ & $\begin{array}{c}\mathcal{E}_{\bar{v}_{e}} \\
{\left[\mathrm{~kg}^{-1} \mathrm{~s}^{-1}\right]}\end{array}$ \\
\hline${ }^{238} \mathrm{U} \rightarrow{ }^{206} \mathrm{~Pb}+8{ }^{4} \mathrm{He}+6 e+6 \bar{v}_{e}$ & 51.7 & 4.47 & 3.26 & $0.95 \times 10^{-4}$ & $7.41 \times 10^{7}$ \\
\hline${ }^{232} \mathrm{Th} \rightarrow{ }^{208} \mathrm{~Pb}+6{ }^{4} \mathrm{He}+4 e+4 \quad \bar{v}_{e}$ & 42.7 & 14.0 & 2.25 & $0.27 \times 10^{-4}$ & $1.63 \times 10^{7}$ \\
\hline${ }^{40} \mathrm{~K} \rightarrow{ }^{40} \mathrm{Ca}+e+\bar{v}_{e}$ & 1.32 & 1.28 & 1.31 & $0.36 \times 10^{-8}$ & $2.69 \times 10^{4}$ \\
\hline
\end{tabular}

$\bar{v}_{e}$ denotes electron anti-neutrinos

Where:

Q is the energy release for the overall decay chain

$\tau_{1 / 2}$ is the half-life of the parent isotope

$\mathrm{E}_{\max }$ is the maximum antineutrino energy in the decay chain

$\varepsilon_{\mathrm{H}}$ is the heating rate, per $\mathrm{kg}$ of the parent isotope

$\varepsilon_{\bar{v}_{e}}$ is the electron antineutrino source rate, per $\mathrm{kg}$ of the parent isotope 
Table $2 \mathrm{U}$, Th and $\mathrm{K}$ global inventories, radiogenic heating and neutrino luminosities according to the Bulk Silicate Earth (BSE) model

\begin{tabular}{cccc}
\hline & $\begin{array}{c}m \\
{\left[10^{17} \mathrm{~kg}\right]}\end{array}$ & $\begin{array}{c}\mathrm{H}_{\mathrm{R}} \\
{\left[10^{12} \mathrm{~W}\right]}\end{array}$ & $\begin{array}{c}\mathrm{L}_{v} \\
{\left[10^{24} \mathrm{~s}^{-1}\right]}\end{array}$ \\
\hline $\mathrm{U}$ & 0.8 & 7.6 & 5.9 \\
$\mathrm{Th}$ & 3.1 & 8.5 & 5.0 \\
${ }^{40} \mathrm{~K}$ & 0.8 & 3.3 & 21.6 \\
\hline
\end{tabular}

As shown in Table 1, the decay chain of ${ }^{238} \mathrm{U}$ into ${ }^{206} \mathrm{~Pb}$ results in 6 anti-neutrinos, one anti-neutrino for each beta decay. Similarly, the decay of ${ }^{232} \mathrm{Th}$ in ${ }^{208} \mathrm{~Pb}$ results in 4 anti-neutrinos. ${ }^{12}$ Because of the neutron inverse $\beta$ decay requires an electron anti-neutrino threshold energy of $1.80 \mathrm{MeV}$, KamLAND cannot detect ${ }^{40} \mathrm{~K}$ anti-neutrinos, but anti-neutrinos from both ${ }^{238} \mathrm{U}$ and ${ }^{232} \mathrm{Th}$ are within the range of this instrument. Additional parameters, including the expected neutrino luminosities, are shown in Table 2.

The overall results of the KamLAND geoneutrino study ${ }^{13}$ show that the sum of the global $U$ and Th inventory is approximately $30 \times 10^{16} \mathrm{~kg}$. Since the global $\mathrm{Th} / \mathrm{U}$ mass ratio is 3.9 , the global U inventory is about $6 \times 10^{16} \mathrm{~kg}$ or $\sim 10 \mathrm{wppb}$ of the mass of the earth. A summary of the KamLAND data is shown in Figure 4, where the geoneutrino signal indicates that the majority of the uranium is in the upper continental crust (UCC) and that relatively little of the inventory is in the oceanic crust, the mantle or the core. The partitioning of the uranium among the upper, middle and lower continental crust and the upper mantle occurs via geochemical processes. ${ }^{14}$ 


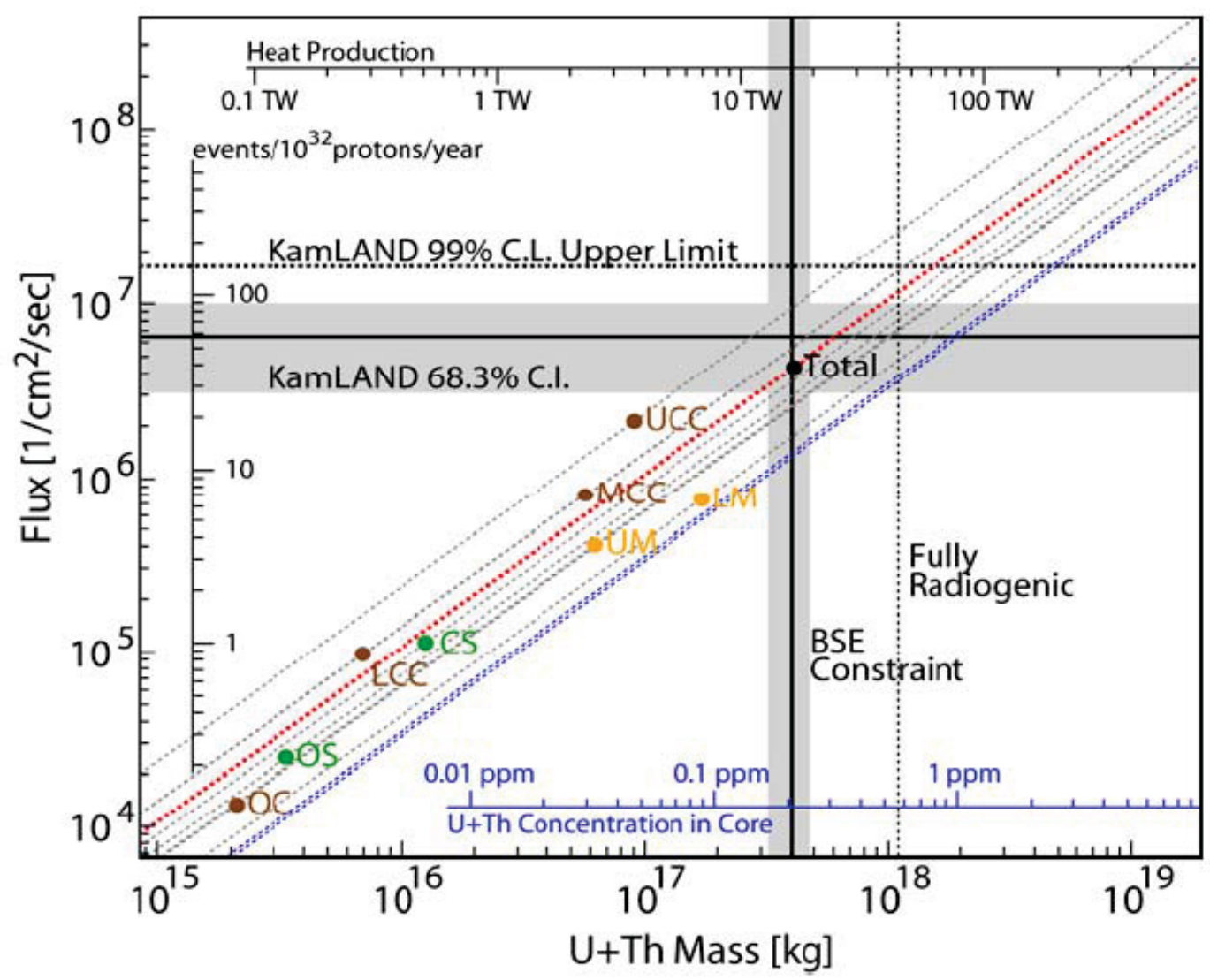

Figure 4. Global U + Th Content based on KAMLAND Geoneutrino Data

The horizontal line is the KamLAND best-fit flux and the horizontal shaded band shows the interval of $68.3 \%$ C.L. The horizontal dotted line is the 99\% C.L. upper limit. The points represent the expected neutrino flux at the KamLAND site in the reference Earth model; Upper Continental Crust (UCC), Middle Continental Crust (MCC), Lower Continental Crust (LCC), Oceanic Crust (OC), Continental Sediment (CS), Oceanic Sediment (OS), Upper Mantle (UM) and Lower Mantle (LM). This assumes a Th/U ratio of 3.9. The blue diagonal lines are response lines of the inner core (lower line) and the outer core (upper line). In the reference model, $U$ and Th amount in the core is set to be zero. The blue auxiliary axis at the bottom indicates the concentration of the $U+T h$ in the core assuming a $T h / U$ ratio of 3.9, for a given $U+T h$ mass in the core. The black auxiliary axis on the top shows heat generation from $U$ and Th for a given U+Th mass. [Ref. 13]

Preliminary results from the newer anti-neutrino detector Borexino at Gran Sasso in the Apennines ${ }^{15}$ generally confirm the KamLAND results but indicate a geoneutrino flux $60 \%$ higher. Because of very low radioactive contamination in the materials of construction for Borexino, a signal to noise ratio of 50:1 was achieved. This greater sensitivity allowed the Borexino researchers to place an upper bound on the power of any critical fissioning zones in the core at $3 \mathrm{TW}$, significantly below the indicated global radiogenic heat production of about $18 \mathrm{TW}$. Collection of geoneutrino data by Borexino is continuing.

Geoneutrino data collected to date indicate that the uranium content of the earth is several orders of magnitude greater than conventional resource estimates. Limited geoneutrino data and an understanding 
of geochemical processes suggest that most of that uranium content is in the upper continental crust. This data provides some confidence that, with further local exploration or advanced extraction technologies, sufficient uranium could be found for several centuries of expanded nuclear power.

\section{Average Vertical Distribution of Uranium and Thorium in the Crust}

As a result of the various igneous processes, the average concentration of uranium is highest at the surface of the continental crust and decreases approximately exponentially with depth.

The anticipated variation of uranium concentration with depth is given by the equation $U(z)=U(z=0) e^{\left(-z / h_{r}\right)}$, where $z$ is the depth in $\mathrm{m}, h_{r}$ is the depth parameter (discussed below) and $U(z)$ is the concentration at depth $z$, in wppm. $U(z=0)$ is the average continental crustal abundance of uranium at the surface, $2.76 \mathrm{wppm}$.

This approximation is based on the presence of heat producing elements, U-238, Th-232 and K-40, in the continental crust, measurements of the thermal conductivity of the crustal materials and the linear temperature distribution with depth measured at many locations. The heat produced in the crust is divided about evenly between U-238 and Th-232, since the crustal abundance mass ratio between Th and $\mathrm{U}$ is 3.9. K-40 is about four orders of magnitudes lower, although potassium has a crustal abundance of $2.1 \%$, since K-40 is only $117 \mathrm{ppm}$ of natural potassium. The thermal energy output of K-40 is about four orders of magnitude below U-238 and Th-232, as shown by Lachenbruch, below. ${ }^{16,17}$ For each of the isotopes shown in Table 3, the thermal output for the entire decay chain (e.g. ${ }^{238} \mathrm{U}$ to ${ }^{206} \mathrm{~Pb}$ and ${ }^{232} \mathrm{Th}$ to ${ }^{208} \mathrm{~Pb}$ ) is given.

Table 3. Sources of Heat in the Upper Continental Crust

\begin{tabular}{|c|c|}
\hline Isotope & thermal output \\
\hline $\mathrm{U}-238$ & $0.095 \mathrm{~mW} / \mathrm{kg}$ \\
\hline $\mathrm{Th}-232$ & $0.027 \mathrm{~mW} / \mathrm{kg}$ \\
\hline $\mathrm{K}-40$ & $3.6 \mathrm{nW} / \mathrm{kg}$ \\
\hline
\end{tabular}

Obviously, this method assumes one-dimensional heat transport and a fairly uniform thermal conductivity, without a significant contribution from flowing fluids. A more recent review by Brady, et al. ${ }^{18}$ provides more details on the technique.

Several measured values of the depth parameter $h_{r}$ are listed in Table 4. ${ }^{19,20}$

Table 4. Temperature Distribution Depth Parameter

\begin{tabular}{|l|c|}
\hline \multicolumn{1}{|c|}{ Location } & $\mathbf{h}_{\mathbf{r}}(\mathbf{m})$ \\
\hline Sierra Nevada & 10,000 \\
\hline Eastern US & 7,500 \\
\hline Norway and Sweden & $7,200 \pm 700$ \\
\hline Eastern Canadian Shield & $7,100 \pm 1700$ \\
\hline Canadian Appalachians & $10,000 \pm 2000$ \\
\hline US Appalachians & $8,100 \pm 1300$ \\
\hline
\end{tabular}

Note: In the other references the depth parameter is denoted $\mathrm{D}$, rather than $\mathrm{h}_{\mathrm{r}}$.

If we assume a depth parameter of $8500 \mathrm{~m}$, based on the above data, then $11 \%$ of the crustal uranium inventory would be expected to be within $1000 \mathrm{~m}$ of the surface and $21 \%$ within $2000 \mathrm{~m}$. Such depths are not economically attractive for open pit or deep shaft mining, though in situ leaching using directional drilling techniques would be feasible. For comparison, the average US natural gas production well in 2008 was $1998 \mathrm{~m}$ deep and some oil/gas wells have exceeded $9000 \mathrm{~m}$. 


\section{Status of laser isotope separation}

The nuclear fuel industry is rapidly moving from gaseous diffusion to gas centrifuge enrichment, as seen in Table 5. Though laser isotope enrichment is not yet operating commercially, the SILEX process, being led by General Electric, is well advanced.

Table 5. Comparison of Enrichment Methods

\begin{tabular}{|l|c|c|c|c|c|}
\hline & & & \multicolumn{3}{|c|}{ Distribution of sources } \\
\hline Enrichment Method & $\begin{array}{c}2012 \text { Est. } \\
\text { Capacity } \\
\text { (MSWU/yr) }\end{array}$ & $\begin{array}{c}\text { Elect. Req'd } \\
\text { (kWhr/SWU) }\end{array}$ & 2000 & 2010 & $\begin{array}{c}2017 \\
\text { (est.) }\end{array}$ \\
\hline Diffusion & 9 & $\sim 2400$ & $50 \%$ & $25 \%$ & 0 \\
\hline Centrifuge & 40 & 50 to 60 & $40 \%$ & $65 \%$ & $93 \%$ \\
\hline Laser & 0 & small & 0 & 0 & $3 \%$ \\
\hline HEU ex weapons & & & $10 \%$ & $10 \%$ & $4 \%$ \\
\hline
\end{tabular}

The SILEX process was developed in Australia, beginning in 1988. In 2007 Silex Systems signed an exclusive agreement with General Electric for commercialization and licensing of the process in Wilmington, NC under the name Global Laser Enrichment (GLE). The present partners in GLE are GE, Hitachi and Cameco. On September 19, 2012 the NRC granted a permit for GLE to build a commercial plant which would enrich uranium to a maximum of $8 \%{ }^{235} \mathrm{U}$. GLE plans to build an initial 1 MSWU/year module and to expand the plant in stages to $6 \mathrm{MSWU} /$ year.

The initial attraction for laser isotope separation is its lower (though proprietary) energy demand. There are, however, other capabilities of LIS that are not often mentioned. Because of the isotope-specific nature of the excitation, LIS is capable of selecting a single middle isotope from a mixture. For instance, LIS could remove the ${ }^{236} \mathrm{U}$ from a mixture of ${ }^{235} \mathrm{U},{ }^{236} \mathrm{U}$ and ${ }^{238} \mathrm{U}$. Niki et al. ${ }^{21}$ have demonstrated the enrichment of natural $\mathrm{Gd}$ to $90 \%{ }^{157} \mathrm{Gd}$ using a combination of lasers for excitation and ionization. The ${ }^{157} \mathrm{Gd}$, with an absorption cross section of $255,000 \mathrm{~b}$, is to be used as a burnable poison in LWRs. And Forsberg ${ }^{22}$ has explored the impact of separating ${ }^{240} \mathrm{Pu}$ for the mixture of plutonium isotopes in used nuclear fuel. While this concept poses several severe technical challenges in the development of the appropriate LIS system and its integration into the reprocessing, the removal of the ${ }^{240} \mathrm{Pu}$ significantly decreases the subsequent production of minor actinides

\section{Conclusion}

Both the increased understanding of the astrophysical origins of the heavy elements and the measurement of geoneutrinos over the last few decades indicate that the overall amount of uranium and thorium in the earth are much larger than current estimates of resources. This conclusion is to be expected, since estimates of resources are based on known deposits of ore concentrations that are economically attractive. Nevertheless, these indications of the global uranium and thorium content can give us confidence that, with sufficient exploration, additional ore bodies can be found as the need and accompanying higher prices appear. Enrichment using laser isotope separation may be less expensive per SWU due to its lower energy requirements. That improvement, in turn, would allow the tails assay to be decreased, thus requiring less natural uranium feed per $\mathrm{kg}$ of fuel. In addition, the unique characteristics of LIS would allow the selective removal of particular isotopes from recycled fuel, avoiding later actinides production. Finally, LIS is being adapted for the tailoring of burnable poisons and structural materials to improve fuel and reactor performance. 


\section{References}

${ }^{1}$ E. Margaret Burbidge, G. R. Burbidge, William A. Fowler and F. Hoyle, "Synthesis of the Elements in Stars," Reviews of Modern Physics, Vol. 29, No. 4, p. 547, Oct. 1957.

${ }^{2}$ Adam Burrows, "Supernova explosions in the Universe," Nature, Vol 403, pp. 727-733, 17 February 2000.

${ }^{3}$ Shinya Wanajo, et al. "The r-Process in Supernova Explosions from the Collapse of O-Ne-Mg Cores," The Astrophysical Journal, 593; 968-979, August 20, 2003.

${ }^{4}$ Harold C. Urey, "The Abundances of the Elements," Physical Review, Vol 88, No. 2, October 15, 1952.

${ }^{5}$ R. A. Alpher, H. Bethe and G. Gamow, "The Origin of Chemical Elements," Physical Review, Vol. 37, No. 7, April 1, 1948.

${ }^{6}$ Harrison S. Brown, "A Table of Relative Abundances of Nuclear Species," Reviews of Modern Physics, Vol 21, No. 4, p 625, October 1949.

${ }^{7}$ Kenneth S. Deffeyes and Ian D. MacGregor, Uranium Distribution in Mined Deposits and in the Earth's Crust. Final Report, GJBX-1(79), Dept of Geological and Geophysical Sciences, Princeton University, Princeton, NJ, prepared for the USDOE, Grand Junction Office, August, 1978.

${ }^{8}$ Shinya Wanajo, et al. "The r-Process in Supernova Explosions from the Collapse of O-Ne-Mg Cores," The Astrophysical Journal, 593; 968-979, August 20, 2003.

${ }^{9}$ Kenneth S. Deffeyes and Ian D. MacGregor, "World Uranium Resources," Scientific American Vol 242, No 1, January 1980, pp. 66-76.

${ }^{10} \mathrm{~T}$. Araki, et al., "Experimental investigation of geologically produced antineutrinos with KamLAND," Nature Vol 436, pp. 499-503 28 July 2005.

${ }^{11}$ C. L Cowan Jr., F. Reines, F. B. Harrison, H. W. Kruse, A. D McGuire, "Detection of the Free Neutrino: a Confirmation," Science, 124 (3212), July 20, 1956.

${ }^{12}$ Gianni Fiorentini, Marcello Lissia, Fabio Mantovani and Riccardo Vannucci, "Geo-Neutrinos: a short review," arXiv:hep-ph/0409152 v1 14 Sep 2004.

${ }^{13}$ S. Enomoto, E. Ohtani, K. Inoue, A. Suzuki, “ Neutrino geophysics with KamLAND and future prospects," arxiv.org/ftp/hep-ph/papers/0508/0508049.pdf

${ }^{14}$ J. Stephen Herring, "Uranium and Thorium Resources," in The Encyclopedia of Energy, Cutler J. Cleveland, editor in chief, Academic Press, 2004.

${ }^{15}$ Borexino Collaboration, “Observation of geo-neutrinos," Physics Letters B 687 (2010) 299-304

${ }^{16}$ Arthur H. Lachenbruch, "Preliminary Geothermal Model of the Sierra Nevada," Journal of Geophysical Research, Vol. 73, No. 22, November 15, 1968.

${ }^{17}$ Arthur H. Lachenbruch, "Crustal Temperature and Heat Production: Implications of the Linear HeatFlow Relation," Journal of Geophysical Research, Vol. 75, No. 17, June 10, 1970. 
${ }^{18}$ Robert J. Brady, Mihai N. Ducea, Steven B. Kidder, Jason B. Saleeby, "The distribution of radiogenic heat production as a function of depth in the Sierra Nevada Batholith, California," Lithos 86 (2006) 229- 244.

19 J. Stephen Herring, "Uranium Resources and Mining," Encyclopedia of Sustainability Science and Technology, pp 11201-11219, Springer Verlag, 2012

${ }^{20}$ Gerald Schubert, Donald Lawson Turcotte and Peter Olson, Mantle Convection in the Earth and Planets, Cambridge University Press, 2001, p. 146.

${ }^{21}$ Hideaki NIKI , Kazunori MOTOKI, Masateru YASUI, Yasunobu HORIUCHI, Shigeki TOKITA \& Yasukazu IZAWA (2006) Selectivity and Efficiency of Laser Isotope Separation Processes of Gadolinium, Journal of Nuclear Science and Technology, 43:4, 427-431

${ }^{22}$ Charles Forsberg "Implications of Plutonium Isotopic Separation on Closed Fuel Cycles and Repository Design," Global 2013; Paper 8622; Salt Lake City, Utah, September 29-October 3, 2013 